\title{
Interactive comment on "The Holocene Evolution of a Sedimentary Carbon Store in a Mid Latitude Fjord" by Craig Smeaton et al.
}

\section{Anonymous Referee \#1}

Received and published: 20 June 2019

The manuscript by Smeaton et al. presents multi-proxy geochemical data from a Scottish fjord spanning the past 8000 yrs. They try to convince that the variability in the terrestrial organic carbon fraction in the fjord represents ecosystem change over land caused by climate reorganization and anthropogenic activity. Essentially, they say that change in vegetation from woodland to heath and grasses increases terrestrial fraction in adjoining fjord but without any references. The present manuscript is speculative at many places, stretches reasoning too far, and lacks references for such reasoning. It is yet not suitable for "Biogeosciences" and requires more work.

Printer-friendly version

Discussion paper

Specific Comments: Comment \#1. The proxy used to determine the terrestrial fraction changes during the Holocene is the $\mathrm{C} / \mathrm{N}$ ratio. Please note that the $\mathrm{C} / \mathrm{N}$ ratio alone in a fjord-setting can provide a wrong impression about the provenance of the organic 
matter. It is so because the $\mathrm{N}$ used in the denominator is the Total Nitrogen that contains both the organic and inorganic nitrogen. In fjords, or wherever there is a large influx of terrestrial supply, a lot of clay bound inorganic nitrogen arrives that skew the C/N ratio (e.g., Schubert \& Calvert, 2001, Deep Sea Res.). To get a correct picture, inorganic nitrogen needs to be removed and the ratio of organic carbon to organic nitrogen needs to be taken. Then the $\mathrm{C} / \mathrm{N}$ ratio will drastically change. Since $\mathrm{C} / \mathrm{N}$ ratio is the major proxy on which this manuscript is based, so Corg/Norg needs to be calculated. Comment \#2. Additionally, $\delta 13 \mathrm{C}$ of the organic matter, which is again a proxy for the provenance, is also required to support the inferences based on the $\mathrm{C} / \mathrm{N}$ ratio. Authors use $\delta 13$ Corg for the last millennium (Fig. 6) but I do not find it anywhere for the Holocene. It needs to be plotted in Fig. 3 and discussed accordingly. Comment \#3. Line 307: The various factors controlling the variability of the mean grain size needs to be mentioned before ascribing it to the enhanced terrestrial input. Comment \#4. Line 310: How a change in the terrestrial ecosystem will increase the proportion of terrestrial organic carbon to the fjord? This sentence (line 310) do not contain any reference to support it. Changing ecosystem can change the type of the terrestrial organic matter but how will it change the amount of the terrestrial organic matter in the fjord? Without references, it stays just speculation and requires a detailed discussion of relevant references. Comment \#5. Line 310-311: No, during the early to mid-Holocene the $\mathrm{C} / \mathrm{N}$ ratio does not remain stable. The $\mathrm{C} / \mathrm{N}$ ratio decreases during early to mid-Holocene. Comment \#6. Line 340-341: This is speculative with no references. Comment \#7. Line 343-345: I appreciate your honesty that you have correctly noted that it is just a speculation as it is not supported by references that change in vegetation from woodland to heath and grasses increases terrestrial fraction in adjoining fjords.

Minor Comments: Comment \#8. Line 134-135: SPG and STG are not shown in Fig. 1. Considering their significance, both should be shown clearly in Fig. 1. Comment \#9. Line 168: What is the reason behind such a huge jump (4 to 6 times) in the sedimentation rate beyond $50 \mathrm{~cm}$ ? Comment \#10. Line 176-179: Please provide details of the standards used to calibrate to the VPDB and Air-N2 scale. Also, provide information

Printer-friendly version

Discussion paper
Interactive comment 
on the precision and number of measurements done to achieve the reported precision. Comment \#11. Line 226: Please mention the number of measurements done to achieve the precision of $0.07 \%$ Comment \#12. Line 256-263: What is the purpose of lines 256 - 263? Authors ascribe all the change in $\delta 180$ values first to temperature and then to salinity, and then reject their assumptions saying it's highly unlikely. Of course, it is. $\mathrm{d} 180$ variability is a function of both the temperature and salinity (and the ice volume effect), which is well known. Ascribing all the $\delta 180$ changes to either salinity or temperature is obviously wrong. So, what's the point in making a wrong assumption and then rejecting it? Comment \#13. Line 265: d180 value is a number and a number can't be enriched or depleted. This is a common misuse in literature and needs to be discontinued. A particular isotope can get enriched or depleted in a material. The correct usage, therefore, is " . . the sample is most depleted in 180 during.... A number can be low or high, negative or positive. So, I suggest replacing "most enriched/depleted $\delta 180$ values" with "highest/lowest $\delta 180$ values" throughout the ms. Comment \#14. Line 267-268: Benthic foraminiferal $\delta 13 \mathrm{C}$ from Ocean Drilling Project Site 980 record is not plotted in Fig. 1. Please do so. Comment \#15. Line 275: Please refer here exact panel in Fig. 3 that shows the expansion of the polar vortex and a shift towards winter-like conditions. Is it $3 \mathrm{a}$ or $3 \mathrm{~b}$ or both or any other panel? Comment \#16. Line 280: When the $\delta 180$ values are lowest i.e., warmest at $5.5 \mathrm{Ka}$, you have highest IRD indicating cold climate and surge in glaciers etc. Explain this discrepancy. Comment \#17. Line 343: OCAR shown as a bar diagram in Fig. 5a needs to be plotted as a Line diagram and should also be included in Fig. 3 below the OC\% and discussed to make the OCAR variability clearer to the readers. Comment \#18. Line 366: Where the crenarcheol concentration is plotted with respect to calibrated age? Comment \#19. Line 367: (i) The end-member values used for calculating the terrestrial fraction are provided in Supplementary Table 3. But also provide the mass balance equations where these particular end-member values are used to arrive at the terrestrial fraction for clarity. (ii) The final marine vs. terrestrial fraction depends a lot on the end-member values, so please explain why only these particular values were chosen? (iii) How the

Printer-friendly version

Discussion paper
Interactive comment

\section{3}


terrestrial fraction will change if Corg/Norg values are taken? Comment \#20. There are several spellings and grammatical mistakes in the manuscript. For example, at many places, "hypothesis" is used in place of "hypothesize". So, the whole manuscript needs to be properly checked for spellings and grammar.

Interactive comment on Biogeosciences Discuss., https://doi.org/10.5194/bg-2019-163, 2019. 